\title{
On the evaluation of the meshing stiffness of external spur gears
}

\author{
Miguel Pleguezuelos, Miryam B. Sánchez, and José I. Pedrero* \\ UNED, Departamento de Mecánica, Juan del Rosal 12, 28040 Madrid, Spain
}

\begin{abstract}
A comparison among different approaches of the meshing stiffness of spur gears has been carried out. The influence of each one on the load sharing, and subsequently on the determinant tooth-root and contact stresses, has also been studied. Equations for the evolution of the meshing stiffness along the path of contact according to all these formulations are also provided.
\end{abstract}

\section{Introduction}

Methods for the evaluation of the load carrying capacity in gear rating standards $[1,2]$ are usually based on simple models of the theory of elasticity -Hertzian contact stress, Navier's bending stress, etc.-, which are applied to the critical load conditions to provide the determinant contact and tooth-root stresses. However, the determinant stresses and critical load conditions depend on the transmitted load at any meshing position, and consequently on the load sharing among couples of teeth in simultaneous contact. As the load sharing is governed by the evolution of the meshing stiffness (MS) along the path of contact, a reliable evaluation of the MS is essential for accurate calculation of the load carrying capacity.

Many studies on the calculation of the MS of spur gear pairs, including analytical methods and finite element (FE) simulations, can be found in literature [36]. All of them result in a symmetric -or almost symmetric- curve of MS respect to the midpoint of the interval of contact, with a maximum at this point. But the procedures, both the definition of the FE models and the development of the numerical calculations, are always tedious and time-consuming. The authors [7-9] presented an approximate equation, of high accuracy and very simple formulation, which expresses the MS as a function of the contact ratio, exclusively. It was useful for strength calculations because the analytic formulation allowed to compute the critical bending and contact stresses by means of simple mathematical procedures [10-13]. The equation was initially obtained from the bending, shear and compressive stiffnesses [7-8]; and it was later modified to consider the Hertzian contact stiffness as well [9].

However, the discussion on the components of the MS is still open. Some authors [3,14] recommend the Weber-Banaschek approach [15] for the contact stiffness, better than the Hertzian one; other authors [16] consider that the gear body stiffness can not be neglected. In this work, different MS approaches, corresponding to combinations of different recommendations for the MS components, are compared. The load sharing ratio (LSR) curves obtained from each approach are also compared and their influence on the determinant stresses are discussed. Values for two coefficients fitting the approximate equation to each MS approach are also provided.

\section{Stiffness components}

The single stiffness is defined as the stiffness of a tooth pair in contact, i.e., the relation between the load at the tooth pair and its deflection. The meshing stiffness at a given contact position will be the sum of the single stiffness of each tooth pair in contact at the given position. The single stiffness $K_{M}$ is the serial composition of several components: the bending stiffness $k_{x}$, the shear stiffness $k_{s}$, and the compressive stiffness $k_{n}$, all of them of both mating teeth; the gear body stiffness $k_{R}$, also in both teeth; and the contact stiffness $k_{H}$. It can be expressed as follows:

$$
\frac{1}{K_{M}}=\left(\frac{1}{k_{x}}+\frac{1}{k_{s}}+\frac{1}{k_{n}}+\frac{1}{k_{R}}\right)_{1}+\left(\frac{1}{k_{x}}+\frac{1}{k_{s}}+\frac{1}{k_{n}}+\frac{1}{k_{R}}\right)_{2}+\frac{1}{k_{H}}
$$

The bending, shear and compressive stiffnesses can be computed from the equations of the linear theory of elasticity. Formulas can be found in [7-9, 14, 15]. Weber and Banaschek [15] developed equations for the gear body stiffness and the contact stiffness, which have been widely used [14]. Nevertheless, the contact stiffness equation has not been completely accepted. It was obtained from the hypothesis of planar deformation state, which implies the presence of a normal stress along the third main direction, which is very unclear. In addition, to integrate the Beltrami's equations, the centrelines of both teeth are assumed to remain undeflected, which is not easy to accept. This results in an overestimation of the contact deflection, which should be smoothed. KissSoft [14], for example, recommends considering the $50 \%$ of the Weber-Banaschek contact deflection for 
Table 1. Components of MS approaches.

\begin{tabular}{|c|c|c|c|}
\hline $\begin{array}{c}\text { Approach } \\
\text { code }\end{array}$ & $\begin{array}{c}\text { Bending } \\
\text { Shear } \\
\text { Compressive }\end{array}$ & Contact & Gear body \\
\hline I & $\mathrm{C}$ & $(-)$ & $(-)$ \\
II & $\mathrm{C}$ & $\mathrm{H}$ & $(-)$ \\
III & $\mathrm{C}$ & $\mathrm{WB}$ & $(-)$ \\
IV & $\mathrm{C}$ & $\mathrm{H}$ & $\mathrm{C}$ \\
V & $\mathrm{C}$ & $\mathrm{WB}$ & $\mathrm{C}$ \\
VI & $\mathrm{C}$ & $50 \% \mathrm{WB}$ & $\mathrm{C}$ \\
\hline
\end{tabular}

C / (-): considered / neglected.

H / WB: Hertzian / Weber-Banaschek approach.

calculations. This reduction results in a MS curve quite similar to that considering the Hertz equation for the contact stiffness, though some authors and researchers consider the Hertzian approach not to be accurate enough to describe the dynamic behaviour of the teeth in contact. Finally, the Weber-Banaschek approach for the gear body stiffness does not account the rim thickness and thus the equation does not describe the MS of thinrimmed gears accurately.

In this work, several approaches for the MS, obtained by combination of different components, are compared. The considered combinations are presented in Table 1. The influence of each one on the prediction of the load sharing is also discussed. Finally, a set of coefficients to fit the approximate equation to each MS approach is provided, including a study of accuracy of the corresponding LSR curves.

\section{Meshing stiffness and load sharing ratio}

The meshing stiffness can be computed from the equations presented in [7-9, 15], and can be expressed as a function of the contact point parameter $\xi$, which describes the meshing position along the path of contact and is defined as [7-9]:

$$
\xi=\frac{z_{1}}{2 \pi} \sqrt{\left(\frac{r_{c 1}}{r_{b 1}}\right)^{2}-1}
$$

where $z$ is the number of teeth, $r_{c}$ the radius of the contact point at the involute profile, $r_{b}$ the base radius, and subscript 1 denotes the pinion. Figure 1 presents the curves of MS according to six considered approaches for a specific spur-gear pair. All of them have a very similar shape, with a maximum at the midpoint of the path of contact, and symmetric branches at both sides of this midpoint. MS curves obtained by FE simulations or experimental tests $[3,14]$ have also the same aspect.

From the values of the MS, the load sharing ratio $R$ can be expressed as:

$$
R_{i}=\frac{F_{i}}{F_{T}}=\frac{K_{M i}}{\sum_{j} K_{M j}}
$$

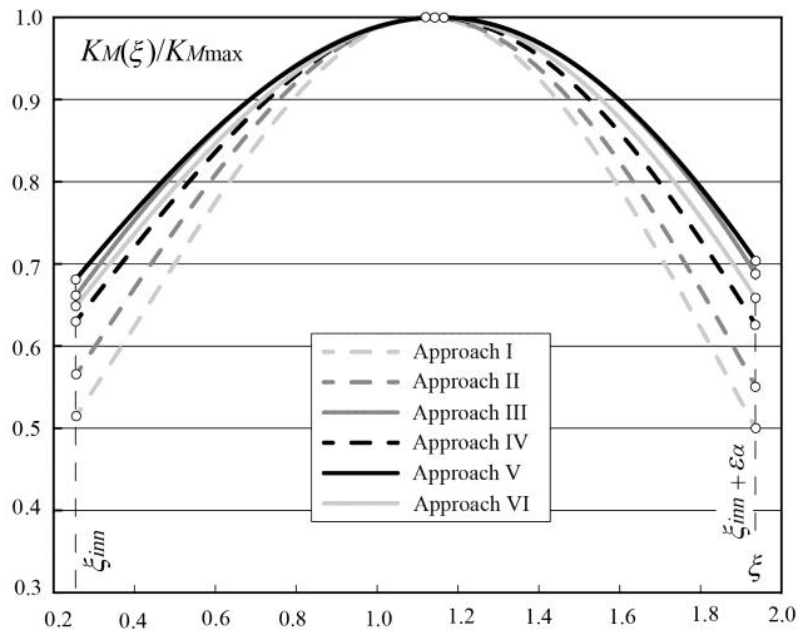

Fig. 1. Curves of MS according to approaches I to VI.

where $F_{i}$ is the load at tooth pair $i, F_{T}$ the total load, and the sum is extended to all the toot pairs in simultaneous contact. If considered $K_{M}(\xi)=0$ outside the interval of contact, the LSR can be also expressed as a function of $\xi$ along the path of contact, namely:

$$
R(\xi)=\frac{F(\xi)}{F_{T}}=\frac{K_{M}(\xi)}{\sum_{j=0}^{z_{1}-1} K_{M}(\xi+j)}
$$

because it can be easily proved that:

$$
\xi_{i+1}=\xi_{i}+1
$$

\subsection{Standard contact ratio spur gears}

Figure 2 presents the curves of LSR corresponding to six considered approaches of MS, for the same specific spur-gear pair of Fig. 1, with contact ratio $\varepsilon_{\alpha}$ between 1 and 2. It is observed that there are non-neglectable differences among the MS approaches: while the LSR along the interval of shared load fluctuates between 0.34 and 0.66 for approach I, for approach V fluctuation occurs between 0.42 and 0.58 . Specifically, the interval of fluctuation is longer for higher values of the MS, and

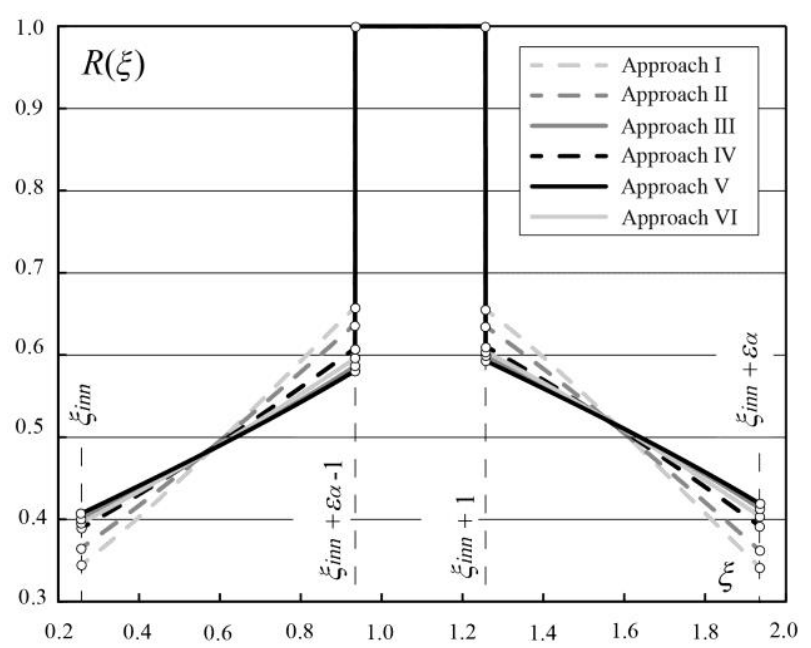

Fig. 2. Curves of LSR according to MS approaches I to VI. 


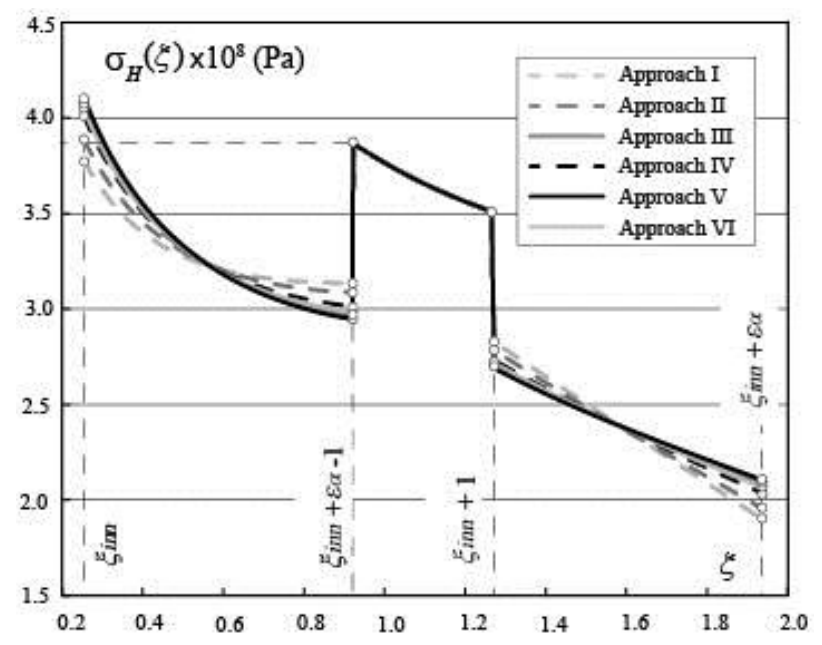

Fig. 3. Determinant contact stress at the inner point of contact.

vice-versa. Obviously, high values of the MS correspond to approach I, while the lowest value correspond to approaches IV, V and VI. Note that diagram in Fig. 1 represents 'normalized' values of the $\mathrm{MS},\left(K_{M} / K_{M \max }\right)$, because according to Eqn. (4) the value of $K_{M \max }$ is not necessary to compute the LSR. However, it is very clear that $K_{M \max -I V}$ or $K_{M \max -\mathrm{V}}$ are smaller than $K_{M \max -\mathrm{I}}$.

These discrepancies do not have significant influence on the determinant stresses and load carrying capacity. The critical load conditions for pitting usually correspond to contact at the inner point of single tooth contact $[1,2,10]$, while for tooth bending, critical load conditions always correspond to contact at the outer point of single tooth contact $[1,2,11]$. In both cases the LSR is equal to 1 for all the approaches.

More significant may be these discrepancies for specific cases in which the determinant contact stress occurs at the inner point of contact; typically for small number of teeth on pinion and high gear ratio. In these cases, discrepancies up to $10 \%$ in the estimation of the nominal contact stress will be obtained. Figure 3 presents the evolution of the contact stress along the path of contact for a spur gear with relatively small number of teeth on pinion (namely, 20) and determinant contact stress at the inner point of contact, computed from six considered approaches. In general, discrepancies will be smaller than $10 \%$ because the most rigid MS approaches (approaches I and II) will shift the critical contact stress to the inner point of single tooth contact, as shown in Fig.3.

\subsection{High contact ratio spur gears}

For spur gears with contact ratio between 2 and 3 -hence forward high contact ratio (HCR) spur gearsdiscrepancies may be greater that that in the previous case. Figure 4 presents the curves of MS according to the considered approaches, for a HCR spur pair with $\varepsilon_{\alpha}=$ 2.12. All of them are quite similar to those in Fig. 1, corresponding to standard contact ratio (SCR) spur pairs.

Nevertheless, discrepancies in the estimation of the LSR are quite more significant. As shown in Fig. 5, differences between approaches I and $\mathrm{V}$ in the estimation

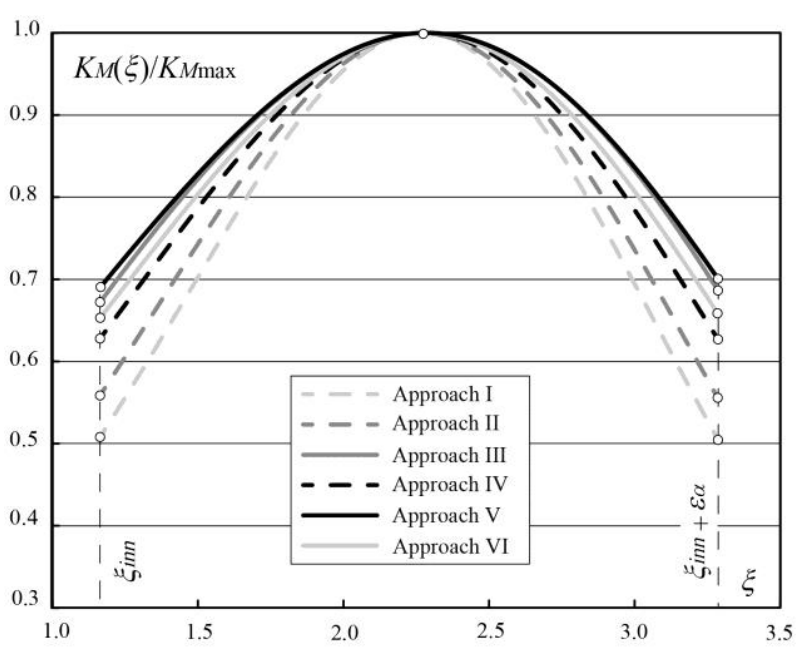

Fig. 4. Curves of MS approaches I to VI for HCR spur gears.

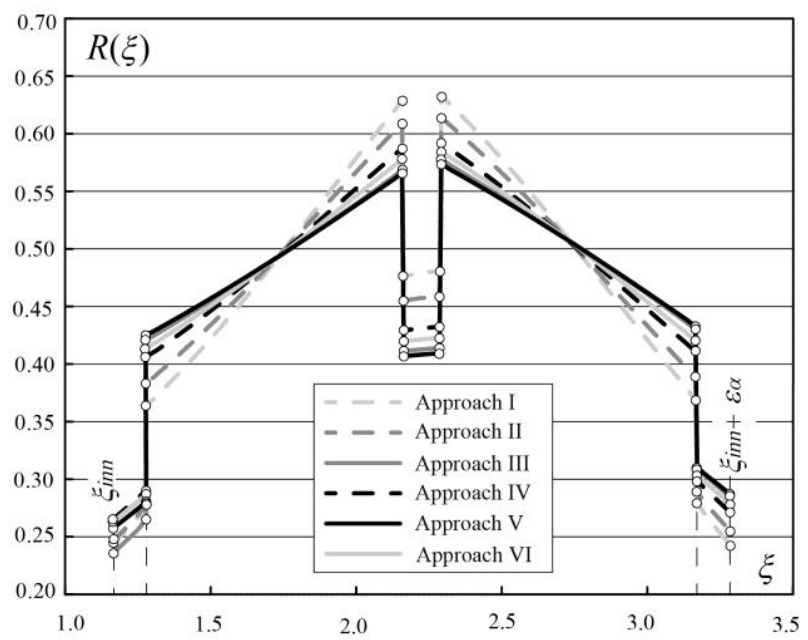

Fig. 5. Curves of LSR for HCR spur gears according to approaches I to VI.

of the LSR at the limits of the intervals of two pair tooth contact are around 0.10 , which means discrepancies of around $20 \%$ in the estimation of the load.

Determinant contact stress in HCR spur gears may arise at the inner point of contact or at the inner limit of the inner interval of two pair tooth contact [12]. In both cases, differences in the estimation of the nominal contact stress will be around $10 \%-12 \%$.

Critical load conditions for bending strength correspond to contact at a point of the outer interval of two pair tooth contact, usually contained between the inner point and the midpoint of the interval [13]. For critical load conditions at the midpoint of the interval, all the MS approaches will provide identical results, as easily derived from Fig. 5. For critical load conditions at the inner point of the interval, discrepancies between approaches I and V may be up to $20 \%$.

The suitability for each approach to describe the MS of a specific spur gear transmission will depend on the material, speed, and geometry of the gear cube. As said above, Approaches I, II and III, which do not consider the gear body stiffness -equivalent to consider rigid body- will be appropriate for solid gears, while approaches IV, V and VI will be more accurate for thinrimmed gears. 
Nevertheless, this discussion is not the aim of this work. Probably, all the approaches will be suitable for specific gearing conditions, thus all of them should be considered for design. However, the calculation of the MS components is always tedious and time-consuming, which becomes a serious problem for repetitive calculations, as usually required in early design stages. In addition, simple formulations of the MS will be useful for optimization studies or complicated calculations of friction power losses, lubrication conditions, etc.

In the next section, analytic approximate equations for each approach of MS are provided. A study of accuracy of each equation is also presented.

\section{Approximate equations for the meshing stiffness}

From Figs. 1 and 4, the evolution of the MS along the path of contact can be accurately represented by a cosine function for all the approaches:

$$
K_{M}(\xi)=K_{M \max } \cos \left[b_{0}\left(\xi-\xi_{m}\right)\right]
$$

where $\xi_{m}$ is the contact point parameter corresponding to the midpoint of the path of contact:

$$
\xi_{m}=\xi_{i n n}+\frac{\varepsilon_{\alpha}}{2}=\xi_{o}-\frac{\varepsilon_{\alpha}}{2}
$$

in which subscripts inn and $o$ denotes the inner and outer points of contact, respectively.

The coefficient $b_{0}$ can be calculated by accounting that the values of the LSR of SCR spur gears, at the inner and outer points of the path of contact -and thus at the inner and outer points of the interval of single tooth contact- are almost uniform for any values of the geometrical parameters of the transmission. Figure 6 shows the values of the LSR at inner and outer points of both the interval of contact and the interval of single tooth contact, for 3775 different combinations of geometrical parameters, according to one of the approaches of the MS. Average values of LSR at singular points of the path of contact for all the approaches are given in Table 2 .

According to Eqns. (3) and (6), the LSR at the outer point of contact can be expressed as follows:

$$
R\left(\xi_{o}\right)=\frac{\cos \left(b_{0} \frac{\varepsilon_{\alpha}}{2}\right)}{\cos \left(b_{0} \frac{\varepsilon_{\alpha}}{2}\right)+\cos \left(b_{0}\left(1-\frac{\varepsilon_{\alpha}}{2}\right)\right)}
$$

If assumed that the cosine can be approximated to the first three terms of the McLaurin series, from Eqn. (8) the coefficient $b_{0}$ can be approximately expressed as:

$$
b_{0}=\left[\frac{1}{2}\left(\frac{\varepsilon_{\alpha}}{2}+\frac{R_{o}}{1-2 R_{o}}\right)^{2}-\frac{R_{o}\left(1-R_{o}\right)}{2\left(1-2 R_{o}\right)^{2}}\right]^{-1 / 2}
$$

where $R_{o}$ is the LSR at the outer point of contact, i.e., $R_{o}$ $=R\left(\xi_{o}\right)$. However, due to the considered approximation

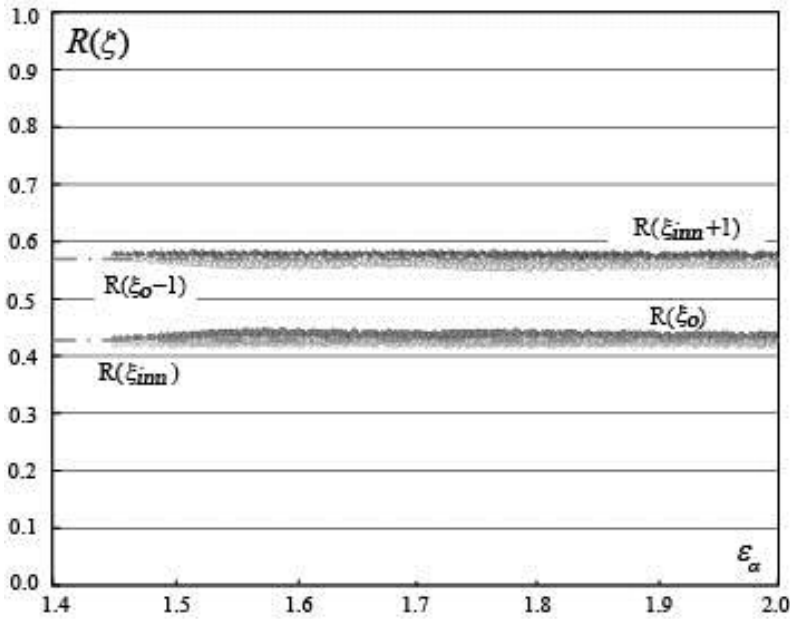

Fig. 6. Values of the LSR at four singular points of the path of contact for SCR spur gears, according to approach V.

Table 2. Average values of LSR at singular points.

\begin{tabular}{|c|c|c|c|c|c|c|}
\hline \multirow{2}{*}{} & \multicolumn{6}{|c|}{ Approach } \\
\cline { 2 - 7 } & I & II & III & IV & V & VI \\
\hline$R\left(\xi_{\text {inn }}\right)$ & 0.338 & 0.361 & 0.419 & 0.388 & 0.421 & 0.404 \\
\hline$R\left(\xi_{o}-1\right)$ & 0.664 & 0.641 & 0.578 & 0.612 & 0.577 & 0.595 \\
\hline$R\left(\xi_{\text {inn }}+1\right)$ & 0.662 & 0.639 & 0.581 & 0.612 & 0.579 & 0.596 \\
\hline$R\left(\xi_{o}\right)$ & 0.336 & 0.359 & 0.422 & 0.388 & 0.423 & 0.405 \\
\hline
\end{tabular}

Table 3. Coefficients $\kappa_{1}$ and $\kappa_{2}$.

\begin{tabular}{|c|c|c|c|c|c|c|}
\hline \multirow{2}{*}{} & \multicolumn{5}{|c|}{ Approach } \\
\cline { 2 - 7 } & I & II & III & IV & V & VI \\
\hline$R_{o}$ & 0.316 & 0.344 & 0.414 & 0.379 & 0.417 & 0.397 \\
\hline$\kappa_{1}$ & 0.86 & 1.11 & 2.40 & 1.56 & 2.50 & 1.92 \\
\hline$\kappa_{2}$ & 0.80 & 1.17 & 4.07 & 2.00 & 4.38 & 2.81 \\
\hline
\end{tabular}

for the cosine function, this value of $b_{0}$ and Eqn. (8) provide a value of $R\left(\xi_{o}\right)$ slightly greater than the initial $R_{o}$, introduced in Eqn. (9). This problem can be solved by introducing in Eqn. (9) an initial $R_{o}$ slightly smaller than $R\left(\xi_{o}\right)$. Table 3 shows the adjusted values of $R_{o}$ used for calculation of $b_{0}$ for six considered approaches, and the obtained values of coefficients $\kappa_{1}$ and $\kappa_{2}$, which are given by:

$$
\begin{gathered}
\kappa_{1}=\frac{R_{o}}{1-2 R_{o}} \\
\kappa_{2}=\frac{R_{o}\left(1-R_{o}\right)}{\left(1-2 R_{o}\right)^{2}}
\end{gathered}
$$

in such a way that: 


$$
b_{0}=\left[\frac{1}{2}\left(\frac{\varepsilon_{\alpha}}{2}+\kappa_{1}\right)^{2}-\kappa_{2}\right]^{-1 / 2}
$$

\section{Accuracy of the approximate equations}

For each approach, the accuracy of the approximate equation has been studied. 3775 different cases have been considered, obtained from the 4000 possible combinations of the following geometrical parameters, but regardless combinations resulting in undercut pinion profile:

- Number of teeth on pinion, $z_{1}: 20,30,40$ and 50.

- Number of teeth on wheel, $z_{2}: 60,70,8090$ and 100.

- Pressure angle, $\alpha_{n}: 18^{\circ}, 19^{\circ}, 20^{\circ}, 21^{\circ}, 22^{\circ}, 23^{\circ}, 24^{\circ}$, $25^{\circ}$.

- Rack shift coefficient on pinion, $x_{1}:-0.1,-0.05,0$, $0.05,0.1$.

- Rack shift coefficient on wheel, $x_{1}:-0.1,-0.05,0$, $0.05,0.1$.

For each combination, the different components of the MS in Eqn. (1) are computed by calculation [7-9, 15] at 101 points of the path of contact. Considering the appropriate components for each approach, the numerical values of the $\mathrm{MS}, K_{M \text {-num }}$, at 101 contact positions are calculated from Eqn. (1). The numerical values of the LSR, $R_{\text {num }}$, at the same 101 contact positions are calculated from Eqn. (4).

Both numerical functions, $K_{M-n u m}$ and $R_{\text {num }}$, have been compared with approximate function of MS given by Eqns. (6), (10) and (11), and approximate function of LSR given by Eqn. (4) with approximate values of $K_{M}$. For each function, MS and LSR, the following parameters have been calculated:

- The maximum error at the inner and outer points of the path of contact.

- The maximum error along the complete path of contact.

- The adjusted $R^{2}$-factor.

The highest maximum errors and the maximum and minimum adjusted $R^{2}$-factor among 3775 considered combinations, are given in Table 4 for approaches I to
VI. These results prove the accuracy of the approximate equations, fully acceptable for strength calculations.

\section{Conclusions}

Different approaches of the meshing stiffness, considering Hertzian or Weber-Banaschek contact stiffness, and accounting or neglecting the gear body stiffness, have been studied. The load sharing ratio and the consequent critical tooth-root and contact stresses, according to each approach, have been compared.

An approximate equation for the meshing stiffness, expressed as a function of the contact ratio exclusively, previously developed by the authors, has been generalized to fit to the other approaches. Values for the changing coefficients for all the considered approaches have been provided.

A study of accuracy of the modified equations has been carried out, revealing very good fits between corresponding numerical meshing stiffness curves, with small estimation errors, and even better fit between load sharing ratio curves.

The procedure to compute the coefficients of the approximate equation for all the approaches is also presented. This procedure would be perfectly valid to calculate new coefficients for any other possible approach, even to approximate meshing stiffness curves obtained from other techniques, as finite element analyses.

\section{Acknowledgments}

The authors express their gratitude to the Spanish Council for Scientific and Technological Research for the support of the project DPI2015-69201-C2-1-R, "Load distribution and strength calculation of gears with modified geometry", as well as the School of Engineering of UNED for the support of the action 2020-MEC25, "Simulation of transmission error in spur gears with profile modification".

\section{References}

1. ISO 6336, parts 1 to 3, International Organization for Standardization, Geneve, Switzerland (2019)

Table 4. Results of accuracy.

\begin{tabular}{|c|c|c|c|c|c|c|c|c|c|c|}
\hline \multirow{3}{*}{ Approach } & \multicolumn{5}{|c|}{ Meshing stiffness } & \multicolumn{5}{|c|}{ Load sharing ratio } \\
\hline & \multicolumn{3}{|c|}{ Highest maximum error } & \multicolumn{2}{|c|}{ Adjusted $R^{2}$-factor } & \multicolumn{3}{|c|}{ Highest maximum error } & \multicolumn{2}{|c|}{ Adjusted $R^{2}$-factor } \\
\hline & $\xi_{\text {inn }}$ & $\xi_{o}$ & {$\left[\xi_{i n n}, \xi_{o}\right]$} & $\max$ & $\min$ & $\xi_{\text {inn }}$ & $\xi_{0}$ & {$\left[\xi_{i n n}, \xi_{o}\right]$} & $\max$ & $\min$ \\
\hline I & 5.88 & 7,02 & 9.39 & 0.9899 & 0.9537 & 3.84 & 3.70 & 4.93 & $>0.9999$ & 0.9932 \\
\hline II & 3.95 & 4.15 & 6.07 & 0.9984 & 0.9721 & 3.22 & 2.26 & 3.79 & $>0.9999$ & 0.9954 \\
\hline III & 5.35 & 4.86 & 5.35 & 0.9999 & 0.9277 & 3.45 & 2.84 & 3.50 & $>0.9999$ & 0.9947 \\
\hline IV & 4.23 & 3.75 & 5.32 & 0.9977 & 0.9619 & 3.01 & 2.30 & 3.63 & $>0.9999$ & 0.9959 \\
\hline $\mathrm{V}$ & 4.69 & 4.56 & 5.01 & 0.9998 & 0.9292 & 3.05 & 3.03 & 3.56 & $>0.9999$ & 0.9946 \\
\hline VI & 4.62 & 3.99 & 4.95 & 0.9994 & 0.9497 & 2.79 & 3.03 & 3.66 & $>0.9999$ & 0.9954 \\
\hline
\end{tabular}


2. ANSI/AGMA 2001-D04, American Gear Manufacturers Association, Alexandría, VA (2004)

3. A. Fernández del Rincón et al., Mech. Mach. Theory 61 (2013)

4. N.L. Pedersen, M.F. Jorgensen, Comput. Struct 135, (2014)

5. H. Ma, J. Zeng, R. Feng, X. Pang, B. Wen, Mech. Mach. Theory 98 (2016)

6. C. Xie, L. Hua, J. Lan, X. Han, X. Wan, X. Xiong, Mech. Syst. Signal Process. 111 (2018)

7. J.I. Pedrero, M. Pleguezuelos, M. Artés, J.A. Antona, Mech. Mach. Theory 45 (2010)

8. M.B. Sánchez, M. Pleguezuelos, J.I. Pedrero, Meccanica 48 (2013)

9. M.B. Sánchez, M. Pleguezuelos, J.I. Pedrero, Mech. Mach. Theory 109 (2017)
10. J.I. Pedrero, M. Pleguezuelos, M. Muñoz, Meh. Mach. Theory 46 (2011)

11. M.B. Sánchez, J.I. Pedrero, M. Pleguezuelos, Int. J. Fatigue 48 (2013)

12. M.B. Sánchez, J.I. Pedrero, M. Pleguezuelos, Mech. Mach. Theory 64 (2013)

13. M.B. Sánchez, M. Pleguezuelos, J.I. Pedrero, Meccanica 49 (2014)

14. B. Mahr, U. Kissling, https://www.kisssoft.ch/english/downloads/pdf/

15. C. Weber, K. Banaschek, Vieweg Verlag, Braunschweig, Germany (1955)

16. P. Sainsot, P. Velex, O. Duverger, J. Mech. Des. 126 (2004) 